\title{
CYCLIC PERIOD CHANGES IN THE ALGOL WW CYGNI
}

\author{
R. T. Zavala, ${ }^{1}$ B. J. McNamara,${ }^{2}$ T. E. Harrison, ${ }^{2}$ H. Bogue, ${ }^{2}$ and H. L. Maness ${ }^{3}$
}

\section{RESUMEN}

Se han observado variaciones cíclicas del período en muchas clases de binarias cercanas con una duración comprendida entre un año y una década. WW Cygni, la binaria tipo Algol, persenta una variación cíclica en su período orbital con una amplitud de algo más de 0.02 días y un período de 56 años. Un tercer o cuarto cuerpo hipotético no explica satisfactoriamente esta variación observada en su período orbital. Las variaciones en luminosidad y color del sistema en el mínimo primario del eclipse concuerdan con el modelo propuesto por Applegate para variaciones de período inducidas por el ciclo magnético en WW Cygni. Hemos comenzado a realizar una serie de observaciones de 9 binarias cercanas para buscar evidencias de variaciones en luminosidad y color consistentes con la hipótesis del ciclo magnético. Se sugiere $\delta$ Librae como un caso apropiado para realizar observaciones con un interferómetro óptico con el fin de verificar la hipótesis del tercer cuerpo propuesta para este sistema tipo Algol.

\section{ABSTRACT}

Year to decade-long cyclic period changes have been observed in many classes of close binaries. The Algol binary WW Cygni shows a cyclic change in its orbital period with an amplitude of slightly more than 0.02 days and a period of 56 years. A hypothetical third or fourth body does not satisfactorily explain the observed variation in the orbital period. The change in luminosity and color of the system at primary eclipse minimum are in agreement with the model proposed by Applegate for a magnetic cycle induced period change in WW Cygni. We have commenced monitoring 9 close binaries for evidence of the luminosity and color changes consistent with the magnetic cycle hypothesis. $\delta$ Librae is suggested as a case suitable for observation with an optical interferometer to test the third body proposed for this Algol system.

Key Words: BINARIES: ECLIPSING — STARS: INDIVIDUAL (WW CYGNI) - STARS: LATE-TYPE

\section{INTRODUCTION}

Long-term cyclic period changes are a fairly common phenomenon in close binary systems. Algol (Hall 1989), RS CVn (Hall \& Kreiner 1980), and W UMa (Hobart et al. 1994) binary classes all exhibit cyclic period changes on decade long timescales. Several hypotheses have been advanced to explain these cyclic period changes. The circular orbits of close binary stars rules out apsidal motion as a possible cause. A third or fourth body could explain these period changes by causing the close binary pair to revolve about the multiple system barycenter. Borkovits \& Hegedüs (1996) carried out a detailed analysis for 18 close binaries of various classes, and found good agreement to the third body hypothesis for four of the systems. A key prediction of the unseen companion argument is that the period variation must be strictly sinusoidal (Kopal 1978).

The interesting result of Hall (1989) that cyclic

\footnotetext{
${ }^{1}$ National Radio Astronomy Observatory, Socorro, New Mexico, USA.

${ }^{2}$ New Mexico State University, Las Cruces, New Mexico, USA.

${ }^{3}$ Grinnell College, Grinnell, Iowa, USA
}

period changes in Algols were restricted to systems in which the secondary star has a convective outer envelope suggests a magnetic origin for the observed period changes. This idea was developed theoretically by Applegate (1992), Lanza, Rodonò, \& Rosner (1998) and Rüdiger et al. (2002). A magnetic activity cycle similar to the solar cycle results in changes to the gravitational quadrupole moment of the distorted, magnetically active star in the close binary system. As the active star progresses through its activity cycle a changing differential rotation modifies the oblateness of the active star, changing the gravitational quadrupole moment. This varying quadrupole moment manifests itself through a cyclically varying orbital period. Large changes in the radius of the active star are ruled out by energetic arguments (Marsh \& Pringle 1990), thus the varying quadrupole moment occurs with a nearly constant radius. The energy which powers the changing differential rotation must come ultimately from the nuclear energy source, and represents a sink for energy that would otherwise be radiated away. This results in a key prediction for the magnetic activity 
TABLE 1

MAGNITUDE AND COLOR CHANGES OF WW CYG

\begin{tabular}{ccc}
\hline Date & $\mathrm{V}$ & $\mathrm{V}-\mathrm{R}$ \\
\hline $1972^{\mathrm{a}}$ & $13.26 \pm 0.01$ & $0.42 \pm 0.02$ \\
$1997-1999^{\mathrm{b}}$ & $13.381 \pm 0.003$ & $0.48 \pm 0.01$ \\
\hline
\end{tabular}

${ }^{a}$ Hall \& Wawrukiewicz, (1972)

${ }^{\mathrm{b}}$ Zavala et al. (2003)

hypothesis, the luminosity and color of a star must vary in phase (Applegate 1992). Such a variation was observed in the RS CVn star CG Cyg by Hall (1991).

\section{PERIOD CHANGES IN WW CYGNI}

WW Cyg is an Algol binary with a period of 3.31 days, ind over a century of eclipse timing data available (Zavala ct, al. 2002, and references therein). This allows the third body hypothesis to be tested over two complete cycles of the period change. Fortunately, WW Cyg also has multi-color photometry near a maxima in the $\mathrm{O}-\mathrm{C}$ curve (Hall \& Wawrukiewicz 1972) which enables a test of the luminosity and color variations of the magnetic activity hypothesis to be made. Zavala et al. (2002) were not able to obtain a satisfactory fit for a third or fourth body, and ruled out an unseen companion as a cause of the cyclic period changes. A preliminary analysis by Zavala et al. (2002) of the color and luminosity of WW Cyg at primary eclipse minima did agree with the predictions of the magnetic activity hypothesis. In Table 1 we present a more quantitative analysis of the luminosity and color variations of WW Cyg at primary eclipse minimum. The $\mathrm{V}$ magnitudes in Table 1 were produced by averaging data points within 0.0066 days of eclipse midpoint. This is just orer $3 \sigma$ of the worst time of minima estimate in Zavala of al. (2002). This yields 35 data points in V obtained from 1997 to 1999 , and 4 data points for Hall \& Wawrukiewicz (1972). Hall \& Mawrukiewicz (1972) used UBV filters, and Zavala et al. (2002) used $V R I$. thus direct comparisons in color index $B-V$ are not possible. The de-reddened $B-V$ of 0.82 of Hall $\&$ Wawrukiewicz (1972) corresponds to a $V-R$ color of 0.42 (Cox 2000 ), or a KOV spectral type. We estimated the error in Hall \& Wawrukiewicz's $V-R$ from the errors presented in their paper. Our estimate of the color of WW Cyg at primary minimum 25 years after Hall \& Wawrukiewicz (1972) is significantly redder, and corresponds to a main sequence spectral type of $\mathrm{K} 2 \mathrm{~V}$.
This agrees with the prediction of Applegate (1992) that the active star should be reddest when it is faintest, and supports a magnetic cycle driven origin for the period changes in WW Cyg.

\section{FUTURE WORK}

Photometric observations of 9 Algol, W UMa, and RS CVn systems are currently underway to test the magnetic activity hypothesis. These systems all show cyclic period variations in their $O-C$ diagrams, and we are obtaining multi-color photometry to examine the correlation between luminosity and color variations as predicted by Applegate. A further interesting case is that of $\delta$ Librae, an Algol binary with a third body suggested by Worek (2001). This third body is predicted to have a mass of $\approx 1 M_{\odot}$ and a period of 2.76 years. The predicted amplitude of the barycenter motion is approximately 5 mas, or 1.8 mas $\mathrm{yr}^{-1}$. The Navy Prototype Optical Interferometer (NPOI, Armstrong et al. 1998) will eventually have $437 \mathrm{~m}$ baselines and a resolution of $0.3 \mathrm{mas}$, and will thus be able to resolve the motion expected by a third body. The superb resolution of optical long baseline interferometry presents a direct method for examining the source of the cyclic period changes in close binaries.

R.T.Z. is grateful to the New Mexico Alliance for Graduate Education and the Professiorate for support to attend this conference. The National Radio Astronomy Observatory is a facility of the National Science Foundation, operated under cooperative agreement by Associated Universities, Inc.

\section{REFERENCES}

Armstrong, J. T. et al. 1998, ApJ, 496, 550

Applegate, J.H. 1992, ApJ, 385, 621

Borkovits, T. \& Hegedüs, T. 1996, A\&AS, 120, 63

Cox, A. N. 2000, "Allen's astrophysical quantities, 4th ed." ed. Arthur N. Cox (New York: AIP Press)

Hall, D. S. 1989, Space Sci. Rev., 50, 219

Hall, D. S. 1991, ApJ, 380, L85

Hall, D. S. \& Kreiner, J.M. 1980, Acta Astron., 30, 287

Hall, D. S. and Wawrukiewicz, A. S. 1972, PASP, 84, 541

Hobart, M. A. et al. 1994, RevMexAA, 28, 111

Kopal, Z. 1978, "Dynamics of close binary systems" (Dordrecht: D. Reidel)

Lanza A.F., Rodonò M. \& Rosner, R. 1998, MNRAS, 296,893

Marsh, T.R. \& Pringle, J.E. 1990, ApJ, 365, 677

Rüdiger et al. 2002, A\&A. 392, 605

Worek, T. F. 2001, PASP, 113, 964

Zavala, R. T. et al. 2002, AJ, 123, 450

Zavala, R. T. et al. 2003, AJ, in preparation 